ERRATA

\title{
ON THE PHYSICAL INTERPRETATION OF THE VILKOVISKY-DE WITT EFFECTIVE ACTION
}

\author{
[MOD. PHY. LETT. A, Vol. 2, No. 11 (1987) 875-886] \\ C. P. BURGESS and G. KUNSTATTER
}

The author wishes the following corrections made:

On Page 886, Ref. 1 should be:

1. J. Schwinger, I. A. S. (Princeton) lectures, unpublished; B. S. De Witt, Relativity, Groups and Topology, ed. by B. S. De Witt and C. De Witt (Gordon and Breach, 1964); G. Jona -Lasinio, Nuovo Cimento 34 (1964) 1790.

The following is added to Ref. 4:

4. B. S. De Witt, in Quantum Gravity 2: A Second Oxford Symposium, ed. by Isham, Penrose and Sciama (Oxford, 1981). 